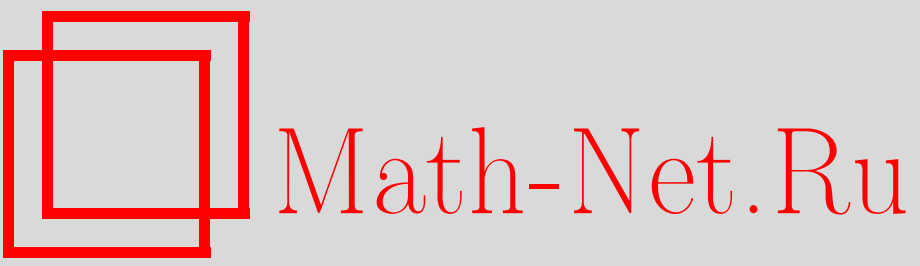

В. Л. Хацкевич, Об условии, обеспечивающем гидродинамическую устойчивость и единственность стационарного и периодического течений жидкости, Итоги науки и техн. Сер. Соврем. мат. и ее прил. Темат. обз., 2021, том 190, 122129

DOI: https://doi.org/10.36535/0233-6723-2021-190-122-129

Использование Общероссийского математического портала Math-Net.Ru подразумевает, что вы прочитали и согласны с пользовательским соглашением

http://www.mathnet.ru/rus/agreement

Параметры загрузки:

IP : 3.85 .73 .92

26 апреля 2023 г., 15:20:30 


\title{
ОБ УСЛОВИИ, ОБЕСПЕЧИВАЮЩЕМ ГИДРОДИНАМИЧЕСКУЮ УСТОЙЧИВОСТЬ И ЕДИНСТВЕННОСТЬ СТАЦИОНАРНОГО И ПЕРИОДИЧЕСКОГО ТЕЧЕНИЙ ЖИДКОСТИ
}

\author{
(c) 2021 г. $\quad$ В. Л. ХАЦКЕВИЧ
}

\begin{abstract}
АннотАция. Предложено условие, обеспечивающее применение первого метода Ляпунова к обоснованию устойчивости стационарных и периодических течений жидкости в ограниченной области, а также единственность решений соответствующих задач.
\end{abstract}

Ключевые слова: эволюционные уравнения Навье-Стокса, гидродинамическая устойчивость, линеаризованная задача, свойство равномерной диссипативности.

\section{ON A CONDITION THAT ENSURES HYDRODYNAMIC STABILITY AND UNIQUENESS OF STATIONARY AND PERIODIC FLUID FLOWS}

\section{(c) $2021 \quad$ V. L. KHATSKEVICH}

\begin{abstract}
In this paper, we propose a condition that ensures the applicability of the first Lyapunov method to justifying stability of stationary and periodic fluid flows in a bounded region and the uniqueness of solutions of the corresponding problems.
\end{abstract}

Keywords and phrases: evolutionary Navier-Stokes equations, hydrodynamic stability, linearized problem, property of uniform dissipativity.

AMS Subject Classification: 35B35, 35Q30

1. Введение. Одним из основных методов изучения устойчивости течения жидкости в настоящее время является метод линеаризации с последующим исследованием спектра линеаризованной задачи (см., напр., $[1,2,6,12])$. В настоящей работе предлагается использовать условие равномерной диссипативности для оператора линеаризованной задачи. Это условие обеспечивает расположение спектра линеаризованной задачи, гарантирующее устойчивость рассматриваемых стационарных и периодических течений в соответствующих пространствах.

Кроме того, в работе установлена единственность решений стационарной и периодической задач для системы уравнений Навье-Стокса в случае, если соответствующая линеаризованная задача обладает свойством равномерной диссипативности. Это придает новый качественный смысл утверждениям о гидродинамической устойчивости. Также в данной работе приведены условия, обеспечивающие равномерную диссипативность для оператора линеаризованной задачи. В последней части статьи показывается применение условия равномерной диссипативности линеаризованного оператора в обосновании принципа усреднения в задаче Навье-Стокса с быстро осциллирующей массовой силой.

2. Устойчивость стационарных течений. Пусть $\Omega$-ограниченная область в $\mathbb{R}^{n}(n=2,3)$ с границей $\partial \Omega$ класса $C^{2}$. В цилиндре $\Omega \times \mathbb{R}_{+}$рассматривается эволюционная система уравнений 
Навье-Стокса:

$$
\begin{gathered}
\frac{\partial \boldsymbol{v}}{\partial t}-\nu \Delta \boldsymbol{v}+\sum_{i=1}^{n} u_{i} \frac{\partial \boldsymbol{v}}{\partial x_{i}}=-\operatorname{grad} p+\boldsymbol{F} \\
\operatorname{div} \boldsymbol{v}=0 \\
\left.\boldsymbol{v}\right|_{\partial \Omega \times \mathbb{R}}=0 \\
\boldsymbol{v}(x, 0)=a(x)
\end{gathered}
$$

Здесь $\boldsymbol{v}$ - вектор скорости, $\nu>0$ характеризует вязкость, $p$ - давление, $\boldsymbol{F}$ - массовая сила. Ниже в этом пункте будем предполагать, что массовая сила $\boldsymbol{F}$ задана, не зависит от времени и квадратично суммируема на $\Omega$.

Пусть система (1)-(4) имеет стационарное решение $\left(\boldsymbol{v}^{0}(x), p^{0}(x)\right)$, т.е. $\left(\boldsymbol{v}^{0}(x), p^{0}(x)\right)$ является решением стационарной задачи:

$$
\begin{gathered}
-\nu \Delta \boldsymbol{v}+\sum_{i=1}^{n} v_{i} \frac{\partial \boldsymbol{v}}{\partial x_{i}}=-\operatorname{grad} p+\boldsymbol{F} \quad \text { в } \Omega, \\
\operatorname{div} \boldsymbol{v}=0 \quad \text { в } \Omega, \\
\boldsymbol{v}=0 \quad \text { на } \partial \Omega .
\end{gathered}
$$

Рассмотрим уравнения Навье-Стокса, линеаризованные в окрестности этого решения:

$$
\begin{gathered}
-\nu \Delta \boldsymbol{v}+\sum_{i=1}^{n} v_{i}^{0} \frac{\partial \boldsymbol{v}}{\partial x_{i}}+\sum_{i=1}^{n} v_{i} \frac{\partial \boldsymbol{v}^{0}}{\partial x_{i}}=-\operatorname{grad} q+\boldsymbol{F}, \\
\operatorname{div} \boldsymbol{v}=0 \quad \text { в } \Omega, \\
\left.\boldsymbol{v}\right|_{\partial \Omega}=0 .
\end{gathered}
$$

Отметим, что разрешимость задач (1)-(4), (5)-(7), (8), (9), а также встречающейся ниже периодической задачи хорошо изучена (см., напр, книги О. А. Ладыженской [3], Ж. Л. Лионса [7], Р. Темама [9]).

Введем необходимые обозначения. Пусть $\boldsymbol{L}^{2}(\Omega)$ - гильбертово пространство векторных квадратично суммируемых функций со скалярным произведением $(\cdot, \cdot)$ и нормой $\|\cdot\|$ :

$$
(\boldsymbol{u}, \boldsymbol{v})=\sum_{j=1}^{n} \int_{\Omega} u_{j} v_{j}^{*} d x, \quad\|\boldsymbol{u}\|=(\boldsymbol{u}, \boldsymbol{u})^{1 / 2}
$$

где знак * означает комплексное сопряжение. Для гладких функций определим скалярное произведение $(\cdot, \cdot)_{1}$ и норму $\|\cdot\|_{1}$ формулами

$$
(\boldsymbol{u}, \boldsymbol{v})_{1}=\sum_{k, j=1}^{n} \int_{\Omega} \frac{\partial u_{j}}{\partial x_{k}} \frac{\partial v_{j}^{*}}{\partial x_{k}} d x, \quad\|\boldsymbol{u}\|_{1}=(\boldsymbol{u}, \boldsymbol{u})_{1}^{1 / 2} .
$$

Через $H$ и $H_{1}$ обозначим замыкание пространства гладких, финитных в $\Omega$ соленоидальных векторных полей по нормам $\|\cdot\|$ и $\|\cdot\|_{1}$, соответственно.

Определим в $H$ оператор $L$, полагая в качестве его области определения $D(L)$ множество соленоидальных, исчезающих на $\partial \Omega$ векторах из $\boldsymbol{W}_{2}^{2}(\Omega)$ :

$$
L \boldsymbol{u} \equiv \Pi\left[-\nu \Delta \boldsymbol{u}+\sum_{i=1}^{n} v_{i}^{0} \frac{\partial \boldsymbol{u}}{\partial x_{i}}+\sum_{i=1}^{n} u_{i} \frac{\partial \boldsymbol{v}^{0}}{\partial x_{i}}\right], \quad \boldsymbol{u} \in D(L),
$$

где $\Pi$ - оператор ортогонального проектирования в $\boldsymbol{L}^{2}(\Omega)$ на подпространство $H$.

Отметим, что между нормами $\|\cdot\|$ и $\|\cdot\|_{1}$ имеет место взаимосвязь

$$
\|\boldsymbol{u}\|_{1}^{2} \geqslant \lambda_{1}\|\boldsymbol{u}\|^{2}, \quad \boldsymbol{u} \in H_{1} .
$$

Здесь $\lambda_{1}$ - первое собственное значение оператора Стокса, действующего в $H$ по формуле $A \boldsymbol{u}=$ $-\Pi \Delta \boldsymbol{u}$ на области определения $D(A)=H_{1} \cap \boldsymbol{W}_{2}^{2}(\Omega)$. 
Ниже будем использовать следующее утверждение (см., например, [12, гл. I, § 5]).

Утверждение 1. Оператор L замкнут, спектр у него чисто точечный: состоит из бесконечной последовательности чисел $\sigma_{1}, \sigma_{2}, \ldots, \sigma_{k}, \ldots$ При этом $\operatorname{Re} \sigma_{k} \rightarrow \infty(k \rightarrow \infty)$.

В [12, гл. 2, § 2] установлена следующая теорема.

Утверждение 2. Пусть спектр оператора L расположен внутри правой полуплоскости:

$$
\operatorname{Re} \sigma(L) \geqslant \sigma_{0}>0
$$

Если область $\Omega$ двумерна, то стационарное решение $\boldsymbol{v}^{0}$ асимптотически устойчиво по Ллпунову в Н. Если область $\Omega-$ трехмернал, то стационарное решение $\boldsymbol{v}^{0}$ асимптотически устойчиво по Ляпунову в $H$.

Отметим, что для трехмерной области $\Omega$ справедлив близкий результат (см. [12]) по устойчивости в пространстве $S_{p}(p \geqslant 3)$, являющимся замыканием гладких соленоидальных, исчезающих на $\partial \Omega$ векторов по норме $\boldsymbol{L}^{p}(\Omega)$.

Нас заинтересовало требование на оператор $L$, которое может обеспечить выполнение условий утверждения 2. Оказалось, что его можно формулировать в терминах диссипативности оператора $L$ (по поводу диссипативных операторов см., например, [8, гл. 1, §6]). В связи с этим введем следующее определение.

Рассматриваемый в гильбертовом пространстве $H$ оператор $L: D(L) \rightarrow H$ называется равномерно к-диссипативным, если выполнено условие

$$
\operatorname{Re}(L \boldsymbol{u}, \boldsymbol{u}) \geqslant \kappa\|\boldsymbol{u}\|^{2}, \quad u \in D(L),
$$

где $\kappa>0$ - фиксированная постоянная.

Имеет место следующее утверждение.

Лемма 1. Если для линеаризованного оператора L, определяемого формулой (11), выполнено условие равномерной к-диссипативности (13) в $H$, то спектр оператора L удовлетворяет соотношению (12) при $\sigma_{0}=\kappa$.

Доказательство. Действительно, пусть $\sigma$ - собственное значение оператора $L$ и $\psi$ - отвечающая ему собственная функция. Тогда согласно (13)

$$
\operatorname{Re}(L \psi, \psi)=\operatorname{Re}(\sigma \psi, \psi)=\operatorname{Re} \sigma\|\psi\|^{2} \geqslant \kappa\|\psi\|^{2} .
$$

Отсюда следует (12) при $\sigma_{0}=\kappa$.

Таким образом, в силу утверждения 2 , справедлива следующая теорема.

Теорема 1. Пусть линеаризованный на $\boldsymbol{v}^{0}$ оператор L равномерно диссипативен. Тогда стационарное решение $\boldsymbol{v}^{0}$ асимптотически устойчиво в пространстве $H$ в случае двумерной области $\Omega$.

Оказывается, диссипативность оператора $L$ обеспечивается малостью нормы $\left\|\boldsymbol{v}^{0}\right\|_{1}$ стационарного решения. Покажем это. Рассмотрим трилинейную форму

$$
b(u, v, w):=\sum_{k, j=1}^{n} \int_{\Omega} u_{k}\left(\frac{\partial v_{j}}{\partial x_{k}}\right) w_{j}^{*} d x, \quad u, v \in H_{1} .
$$

Мы будем использовать модификацию известного свойства трилинейной формы [9, гл. $2, \S 11]$, а именно, для комплекснозначных функций $u, v$ справедливо равенство

$$
\operatorname{Re} b(\boldsymbol{v}, \boldsymbol{u}, \boldsymbol{u})=0, \quad \boldsymbol{u}, \boldsymbol{v} \in H_{1} .
$$

Кроме того, известно соотношение

$$
|b(\boldsymbol{u}, \boldsymbol{v}, \boldsymbol{w})| \leqslant C\|\boldsymbol{v}\|_{1}\|\boldsymbol{u}\|_{1}\|\boldsymbol{w}\|_{1}, \quad \boldsymbol{u}, \boldsymbol{v}, \boldsymbol{w} \in H_{1}
$$

(см. [9, гл. $2, \S 11]$ ), где постоянная $C$ зависит от области $\Omega$. 
Заметим, что для стационарного решения $\boldsymbol{v}^{0}$ задачи (2)-(7) справедливо соотношение

$$
\nu\left(\boldsymbol{v}^{0}, \boldsymbol{v}\right)_{1}+b\left(\boldsymbol{v}^{0}, \boldsymbol{v}^{0}, \boldsymbol{v}\right)=(\boldsymbol{F}, \boldsymbol{v}), \quad \boldsymbol{v} \in H_{1},
$$

т.е. $\boldsymbol{v}^{0}$ - слабое решение.

Из (16) при $\boldsymbol{v}=\boldsymbol{v}^{0}$ в силу (14) следует оценка

$$
\left\|\boldsymbol{v}^{0}\right\|_{1} \leqslant \frac{1}{\nu \sqrt{\lambda_{1}}}\|\boldsymbol{F}\| .
$$

Теорема 2. Если выполнено соотношение

$$
\nu-\frac{c}{\nu \sqrt{\lambda_{1}}}\|\boldsymbol{F}\|>0
$$

то оператор L, линеаризованный на $\boldsymbol{v}^{0}$, равномерно диссипативен при

$$
\kappa=\lambda_{1}\left(\nu-\frac{c}{\nu \sqrt{\lambda_{1}}}\|\boldsymbol{F}\|\right) .
$$

Доказательство. В силу определения оператора $L$ и с учетом (14) имеем

$$
\operatorname{Re}(L \boldsymbol{u}, \boldsymbol{u})=-\nu\|\boldsymbol{u}\|_{1}^{2}+\operatorname{Re} b\left(\boldsymbol{v}^{0}, \boldsymbol{u}, \boldsymbol{u}\right)+\operatorname{Re} b\left(\boldsymbol{u}, \boldsymbol{v}^{0}, \boldsymbol{u}\right)=-\nu\|\boldsymbol{u}\|_{1}^{2}+\operatorname{Re} b\left(\boldsymbol{u}, \boldsymbol{v}^{0}, \boldsymbol{u}\right) .
$$

При этом согласно (15), (17)

$$
\operatorname{Re} b\left(\boldsymbol{u}, \boldsymbol{v}^{0}, \boldsymbol{u}\right) \leqslant\left|b\left(\boldsymbol{u}, \boldsymbol{v}^{0}, \boldsymbol{u}\right)\right| \leqslant C\left\|\boldsymbol{v}^{0}\right\|_{1}\|\boldsymbol{u}\|_{1}^{2} \leqslant C \frac{\|F\|}{\nu \sqrt{\lambda_{1}}}\|\boldsymbol{u}\|_{1}^{2} .
$$

Тогда

$$
\operatorname{Re}(L \boldsymbol{u}, \boldsymbol{u}) \geqslant\left(\nu-\frac{\|F\|}{\nu \sqrt{\lambda_{1}}}\right)\|\boldsymbol{u}\|_{1}^{2},
$$

что и обеспечивает справедливость утверждения.

Согласно (17) малость нормы $\left\|\boldsymbol{v}^{0}\right\|_{1}$ обеспечена, если вязкость $\nu>0$ достаточно велика, либо массовая сила $F$ мала по норме $L_{2}$.

Но при этом, как известно (см. [9, гл. II, § 1]), стационарное решение единственно. Это наводит на мысль, что имеет место следующее утверждение.

Теорема 3. Пусть $\boldsymbol{v}^{0}$ - решение стационарной задачи (5)-(7), а соответствующий ему линеаризованный оператор L, определяемый равенством (11), обладает свойством равномерной диссипативности (13). Тогда $\boldsymbol{v}^{0}$ является единственным решением стационарной задачи (5)(7).

Доказательство. Пусть $\boldsymbol{v}^{0}$ - решение стационарной задачи (5)-(7). Тогда оно удовлетворяет соотношению (16). Пусть $\boldsymbol{u}^{0}$ - некоторое другое решение задачи (16). Положим $\boldsymbol{u}=\boldsymbol{v}^{0}-\boldsymbol{u}^{0}$. Вычтем друг из друга уравнения (16), соответствующие $\boldsymbol{v}^{0}$ и $\boldsymbol{u}^{0}$. Тогда получим соотношение

$$
\nu(\boldsymbol{u}, \boldsymbol{v})_{1}+b\left(\boldsymbol{v}^{0}, \boldsymbol{u}, \boldsymbol{v}\right)+b\left(\boldsymbol{u}, \boldsymbol{v}^{0}, \boldsymbol{v}\right)-b(\boldsymbol{u}, \boldsymbol{u}, \boldsymbol{v})=0, \quad \boldsymbol{v} \in V .
$$

Полагая в $(19) \boldsymbol{v}=\boldsymbol{u}$ и используя свойство (14) для вещественнозначных функций, находим

$$
\nu(\boldsymbol{u}, \boldsymbol{u})_{1}+b\left(\boldsymbol{v}^{0}, \boldsymbol{u}, \boldsymbol{u}\right)+b\left(\boldsymbol{u}, \boldsymbol{v}^{0}, \boldsymbol{u}\right)=0 .
$$

С другой стороны, по определению оператора $L$ левая часть этого равенства равна $(L \boldsymbol{u}, \boldsymbol{u})$. Тогда в силу предположения о равномерной диссипативности (13) оператора $L$ и вещественнозначности функции $\boldsymbol{u}$ имеем $(L \boldsymbol{u}, \boldsymbol{u})=\operatorname{Re}(L \boldsymbol{u}, \boldsymbol{u}) \geqslant \kappa\|\boldsymbol{u}\|^{2}$. Следовательно, $0 \geqslant \kappa\|\boldsymbol{u}\|^{2}$, т.е. $\boldsymbol{u}=0$, что и требовалось доказать.

Отметим, что на самом деле результат теоремы 3 справедлив, если предполагать, что $\boldsymbol{v}^{0}-$ только слабое решение, т.е. удовлетворяет (16).

Вообще, вопрос о единственности решений стационарной задачи открыт до настоящего времени. Известны случаи неединственности близких задач (результаты Юдовича, Рабиновича, Вельте и др., см., например, [9, гл. II, § 4]. Единственность решения стационарного уравнения Навье- 
Стокса (5)-(7) установлена лишь в предположении, что $\nu$ достаточно велико или что заданные силы достаточно малы (см., например, [9, гл. II, § 1]).

Поэтому вопрос, какие дополнительные условия обеспечивают единственность, весьма актуален. В нашем случае это условие равномерной диссипативности линеаризованного оператора. Теорема 3 придает новый качественный смысл теореме 1 о гидродинамической устойчивости, поскольку в условиях теоремы 1 решение стационарной задачи единственно по теореме 3.

3. Устойчивость периодических течений. Рассмотрим задачу о вынужденных колебаниях жидкости в ограниченной области с фиксированным периодом $T>0$.

Пусть $\Omega-n$-мерная $(n=2,3)$ ограниченная область с границей $\partial \Omega$ класса $C^{2}$. В бесконечном цилиндре $\Omega \times \mathbb{R}$ рассмотрим задачу о $T$-периодических решениях системы Навье-Стокса (1)(3). Ниже предполагается, что массовая сила $\boldsymbol{F}=\boldsymbol{F}(t, x)$ является $T$-периодической по $t$, а при каждом фиксированном $t$ квадратично суммируема по $x \in \Omega$.

Пусть $\boldsymbol{v}^{0}(t)$ - некоторое $T$-периодическое решение задачи (1)-(3). Аналогично (11) на области определения $D(L)=H_{1} \cap \boldsymbol{W}_{2}^{2}(\Omega)$ рассмотрим оператор $L(t)$, определяемый формулой

$$
L(t) \boldsymbol{z} \equiv \Pi\left(-\nu \Delta \boldsymbol{z}+\sum_{i=1}^{n} v_{i}^{0}(t) \frac{\partial \boldsymbol{z}}{\partial x_{i}}+\sum_{i=1}^{n} z_{i} \frac{\partial \boldsymbol{v}^{0}}{\partial x_{i}}\right) .
$$

Согласно [12, гл. III, § 3] в $H$ рассматривается линеаризованное на $\boldsymbol{v}^{0}(t)$ однородное уравнение

$$
\frac{d \boldsymbol{z}}{d t}+L(t) \boldsymbol{z}=0
$$

Решение уравнения (21) ищется в виде

$$
\boldsymbol{z}(t)=e^{\sigma t} \boldsymbol{w}(t)
$$

с $T$-периодической по $t$ вектор-функцией $\boldsymbol{w}(t)$.

Комплексные значения параметра $\sigma$, для которых уравнение $(21)$ имеет ненулевые решения вида (22) ищут, решая спектральную задачу

$$
\frac{d \boldsymbol{w}}{d t}+L(t) \boldsymbol{w}+\sigma \boldsymbol{w}=0, \quad \boldsymbol{w}(t+T)=\boldsymbol{w}(t) .
$$

Совокупность всех таких $\sigma$ называется спектром устойчивости основного течения $\boldsymbol{v}^{0}(t)$ и обозначается $\mathfrak{S}$.

Утверждение 3 (см. [12, гл. III, § 3]). Спектр устойчивости $\mathfrak{S}$ состоит из конечнократных собственных значений.

Утверждение 4 (см. [12, гл. III, § 3]). Пусть спектр устойчивости периодического решения $\boldsymbol{v}^{0}(t)$ лежит внутри левой полуплоскости, т.е. для всех собственных значений задачи (23) справедливо неравенство $\operatorname{Re} \sigma \leqslant-\sigma_{0}<0$. Тогда течение $\boldsymbol{v}^{0}(t)$ асимптотически устойчиво по Ляпунову в пространстве $H_{1}$.

Укажем условие, обеспечивающее выполнение предположений утверждения 4.

Лемма 2. Пусть при каждом $t$ для линеаризованного оператора $L(t)$, определяемого формулой (20), выполнено условие равномерной к-диссипативности (13). Тогда спектр задачи (23)

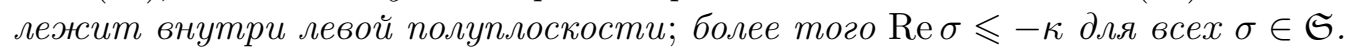

Доказательство. Предположим противное, т.е. пусть найдется такое $\sigma_{0} \in \mathfrak{S}$, что $\operatorname{Re} \sigma_{0}>-\kappa$. Положим $\gamma=\kappa+\operatorname{Re} \sigma_{0}$. По определению $\gamma>0$. Рассмотрим оператор $B(t)=L(t)+\sigma_{0} I$, где $I-$ единичный оператор в $H$. Тогда согласно условию (13) и сделанному предположению имеем

$$
\operatorname{Re}(B(t) \boldsymbol{u}, \boldsymbol{u})=\operatorname{Re}(L(t) \boldsymbol{u}, \boldsymbol{u})+\operatorname{Re} \sigma_{0}(\boldsymbol{u}, \boldsymbol{u}) \geqslant \gamma\|\boldsymbol{u}\|^{2}, \quad \boldsymbol{u} \in D(L) .
$$

Как известно (см., например, [10, гл. II, § 2.1]), условие (24) обеспечивает единственность $T$ периодического решения уравнения $(23)$. Следовательно, $\boldsymbol{w}(t) \equiv 0$. Таким образом, $\sigma_{0}$ не входит в спектральное множество $\mathfrak{S}$. Полученное противоречие доказывает лемму 2.

Из утверждения 4 и леммы 2 вытекает следующая теорема. 
Теорема 4. Пусть при каждом $t$ для линеаризованного оператора $L(t)$, определяемого формулой (20), выполнено условие равномерной к-диссипативности (13). Тогда периодическое течение $\boldsymbol{v}^{0}(t)$ асимптотически устойчиво по Ляпунову в пространстве $H_{1}$.

Кроме того, условие (13) на $L(t)$ обеспечивает единственность $T$-периодического решения задачи (1)-(3).

Вопрос о единственности в общей ситуации, по-видимому, до сих пор остается открытым. Единственность решения задачи (19), (20) установлена в предположении «малости» неоднородности $\boldsymbol{F}$, в частности, при достаточной малости $\|\boldsymbol{F}\|_{L^{\infty}(0, T ; H)}$ (см. [6, с. 498]). В нашей ситуации имеет место следующая теорема.

Теорема 5. Пусть $\boldsymbol{v}^{0}(t)$ есть Т-периодическое решение задачи (1)-(3). Пусть при каждом $t$ для линеаризованного на $\boldsymbol{v}^{0}(t)$ оператора $L(t)$ выполнено условие равномерной $\kappa$-диссипативности (13). Тогда $\boldsymbol{v}^{0}(t)$ является единственным Т-периодическим решением задачи (1)-(3).

Доказательство. Заметим, что $\boldsymbol{v}^{0}(t)$ удовлетворяет соотношению (слабое решение)

$$
\left(\frac{d}{d t} \boldsymbol{v}^{0}, \boldsymbol{v}\right)+\nu\left(\boldsymbol{v}^{0}, \boldsymbol{v}\right)_{1}+b\left(\boldsymbol{v}^{0}, \boldsymbol{v}^{0}, \boldsymbol{v}\right)=(\boldsymbol{F}, \boldsymbol{v}), \quad \boldsymbol{v} \in H_{1}
$$

Пусть $\boldsymbol{u}^{0}$ - некоторое другое $T$-периодическое решение задачи (1)-(3). Положим $\boldsymbol{u}=\boldsymbol{v}^{0}-\boldsymbol{u}^{0}$. Вычтем друг из друга уравнения (25), соответствующие $\boldsymbol{v}^{0}$ и $\boldsymbol{u}^{0}$. Тогда получим соотношение

$$
\left(\frac{d}{d t} \boldsymbol{u}, \boldsymbol{v}\right)+\nu(\boldsymbol{u}, \boldsymbol{v})_{1}+b\left(\boldsymbol{u}, \boldsymbol{v}^{0}, \boldsymbol{v}\right)+b\left(\boldsymbol{v}^{0}, \boldsymbol{u}, \boldsymbol{v}\right)-b(\boldsymbol{u}, \boldsymbol{u}, \boldsymbol{v})=0, \quad \boldsymbol{v} \in H_{1} .
$$

Полагая здесь $\boldsymbol{v}=\boldsymbol{u}$ и используя (14) для вещественнозначных функций, получим

$$
\frac{1}{2} \frac{d}{d t}\|\boldsymbol{u}(t)\|^{2}+\nu\|\boldsymbol{u}(t)\|_{1}^{2}+b\left(\boldsymbol{u}, \boldsymbol{v}^{0}, \boldsymbol{u}\right)=0 .
$$

Согласно определению оператора $L(t)$ и (14) это равенство можно переписать в виде

$$
\frac{1}{2} \frac{d}{d t}\|\boldsymbol{u}(t)\|^{2}+(L(t) \boldsymbol{u}, \boldsymbol{u})=0
$$

Отсюда в силу равномерной $\kappa$-диссипативности оператора $L(t)(13)$ и вещественнозначности функции $u(t)$ получим

$$
\frac{d}{d t}\|\boldsymbol{u}(t)\|^{2} \leqslant-2 \kappa\|\boldsymbol{u}(t)\|^{2}
$$

Тогда при всех $\tau \in \mathbb{R}$ имеем

$$
\|\boldsymbol{u}(t)\|^{2} \leqslant e^{-2 \kappa(t-\tau)}\|\boldsymbol{u}(\tau)\|^{2}, \quad t \geqslant \tau .
$$

Устремляя в (26) $\tau \rightarrow-\infty$ и используя периодичность (а значит, ограниченность функции $\boldsymbol{u}(\tau)$ ), убедимся, что $\boldsymbol{u}(t) \equiv 0$.

Следствие. Для разности решений $\boldsymbol{v}^{0}$ и $\boldsymbol{v}$ задачи (1)-(3) справедлива оченка

$$
\left\|\boldsymbol{v}^{0}(t)-\boldsymbol{v}(t)\right\| \leqslant e^{-\kappa t}\left\|\boldsymbol{v}^{0}(0)-\boldsymbol{v}(0)\right\|, \quad t \geqslant 0 .
$$

Оценка (27) гарантирует экспоненциальную асимптотическую устойчивость (конвергентность) $T$-периодического решения $\boldsymbol{v}^{0}(t)$.

Отметим, что аналогично теореме 2 справедливо утверждение об условиях, обеспечивающих равномерную $\kappa$-диссипативность оператора $L(t)$. При этом используются оценки периодических решений задачи (1)-(3) (см., например, [11]). Можно показать, что (в частности) условие малости $\|\boldsymbol{F}\|_{L^{\infty}(0, T ; H)}$ обеспечивает равномерную диссипативность оператора $L(t)$. 
4. О принципе усреднения в периодической задаче для системы уравнений Навье-Стокса. Условие равномерной диссипативности линеаризованного оператора полезно также и при обосновании метода усреднения в задачах с быстро осциллирующей массовой силой. Опишем этот класс задач (см. $[2,4,5,11])$.

В бесконечном цилиндре $\Omega \times R$ рассматривается задача о $T / \omega$-периодическом решении

$$
\begin{gathered}
\frac{\partial \boldsymbol{u}}{\partial t}-\nu \Delta \boldsymbol{u}+\sum_{i=1}^{n} u_{i} \frac{\partial \boldsymbol{u}}{\partial x_{i}}+\operatorname{grad} p=\boldsymbol{f}(x, \omega t), \quad \operatorname{div} \boldsymbol{u}=0, \\
\left.\boldsymbol{u}\right|_{\partial \Omega \times \mathbb{R}}=0 \\
\boldsymbol{u}(x, 0)=\boldsymbol{u}(x, T / \omega) .
\end{gathered}
$$

Здесь $T>0$ - фиксированное число, $\omega>0$ - «большой» параметр осцилляции. При этом предполагается, что функция $\boldsymbol{f}(x, \tau)-T$-периодична по второму аргументу.

Наряду с (28)-(30) в $\Omega$ рассматривается усредненная стационарная задача

$$
\begin{gathered}
-\nu \Delta \boldsymbol{u}+\sum_{i=1}^{n} u_{i} \frac{\partial \boldsymbol{u}}{\partial x_{i}}=\overline{\boldsymbol{f}}-\operatorname{grad} q, \quad \operatorname{div} \boldsymbol{u}=0 . \\
\left.\boldsymbol{u}\right|_{\partial \Omega \times \mathbb{R}}=0,
\end{gathered}
$$

где в правой части фигурирует среднее значение $\bar{f}$ функции $\boldsymbol{f}(x, \tau)$, задаваемое формулой

$$
\overline{\boldsymbol{f}}(x)=\frac{1}{T} \int_{0}^{T} \boldsymbol{f}(x, \tau) d \tau
$$

Пусть $\boldsymbol{u}^{0}$ - некоторое решение задачи (31), (32). Рассмотрим линеаризованную на $\boldsymbol{u}^{0}$ задачу

$$
\begin{gathered}
-\nu \Delta \boldsymbol{u}+\sum_{i=1}^{n} u_{i}^{0} \frac{\partial \boldsymbol{u}}{\partial x_{i}}+\sum_{i=1}^{n} u_{i} \frac{\partial \boldsymbol{u}^{0}}{\partial x_{i}}+\operatorname{grad} q=\overline{\boldsymbol{f}}, \quad \operatorname{div} \boldsymbol{u}=0 \text { в } \Omega, \\
\int_{\Omega} q d x=0,\left.\quad \boldsymbol{u}\right|_{\partial \Omega}=0 .
\end{gathered}
$$

Определим в пространстве $H$ линейный оператор $A_{0}$, соответствующий задаче $(33),(34)$ :

$$
A_{0} \boldsymbol{z} \equiv \Pi\left[-\nu \Delta \boldsymbol{z}+\sum_{i=1}^{n} u_{i}^{0} \frac{\partial \boldsymbol{z}}{\partial x_{i}}+\sum_{i=1}^{n} z_{i} \frac{\partial \boldsymbol{u}^{0}}{\partial x_{i}}\right] \boldsymbol{z} \in H_{1} \cap \boldsymbol{W}_{2}^{2}(\Omega),
$$

где П - проектор в $L_{2}(\Omega)$ на подпространство $H$ соленоидальных векторных полей.

Сформулируем в удобном для нас виде результат из [5, гл. XI, § 5] (см. также $[2,4,5,11])$.

Утверждение 5. Пусть $\Omega$-ограниченная область пространства $\mathbb{R}^{n}, n=2,3$, граница $\partial \Omega$ области класса $C^{2}$, неоднородность $\boldsymbol{f}(x, \tau)$ принадлежсит классу $C(0, T ; H)$. Пусть $\boldsymbol{u}^{0}$ - некоторое решение задачи (31), (32) и спектр линейного оператора (35) не содержст точек мнимой оси. Тогда найдутся такие значение параметра осиилляиии $\omega_{0}>0$ и положстельное число $r$, что при $\omega>\omega_{0}$ задача (28)-(30) имеет единственное в шаре $\left\|\boldsymbol{u}^{0}-\boldsymbol{u}\right\|_{1} \leqslant r$ (возможно, обобщенное) решение $\boldsymbol{u}^{\omega}$. При этом $\left\|\boldsymbol{u}^{\omega}-\boldsymbol{u}^{0}\right\|_{1} \rightarrow 0$ при $\omega \rightarrow+\infty$.

В силу леммы 1 и теоремы5 имеет место следующее утверждение.

Теорема 6. Пусть оператор $A_{0}$, определяемый формулой (35), равномерно диссипативен. Тогда справедливо заключение утверждения 5. При этом решение $\boldsymbol{u}^{0}$ задачи (31), (32) единственно.

Отметим, что единственность $\boldsymbol{u}^{0}$ придает новый качественный смысл обоснованию метода усреднения. 


\section{СПИСОК ЛИТЕРАТУРЫ}

1. Джозеф Д. Устойчивость движений жидкости. - М., 1981.

2. Копачевский Н. Д., Крейн С. Г., Нго Зуй Кан Операторные методы в линейной гидродинамике: Эволюционные и спектральные задачи. - М.: Наука, 1989.

3. Ладыженскал О. А. Математические вопросы динамики вязкой несжимаемой жидкости. - М.: Наука, 1970.

4. Левенштам В. Б. Асимптотическое интегрирование системы Навье-Стокса с быстро осциллирующей массовой силой// Диффер. уравн. - 2001. - 37, № 5. - С. 696-705.

5. Левитан Б. М., Жиков В. В. Почти периодические функции и дифференциальные уравнения. - М.: МГУ, 1978.

6. Линъ Цзя-цзяло Теория гидродинамической устойчивости. - М., 1958.

7. Лионс Ж. Л. Некоторые методы решения нелинейных краевых задач. - М.: Мир, 1972.

8. Симоненко И. Б. Метод усреднения в теории нелинейных уравнений параболического типа с приложением к задачам гидродинамической устойчивости. - Ростов-на-Дону: Изд-во Ростов. ун-та, 1989.

9. Темам Р. Уравнения Навье-Стокса. Теория и численный анализ. - М.: Мир, 1981.

10. Трубников Ю. А., Перов А. И. Дифференциальные уравнения с монотонными нелинейностями. Наука и техника, 1986.

11. Хачкевич В. Л. О принципе усреднения в периодической по времени задаче для уравнений Навье-Стокса с быстро осциллирующей массовой силой// Мат. заметки. - 2016. - 99, № 5. - С. 764-777.

12. Юдович В. И. Метод линеаризации в гидродинамической теории устойчивости. - Ростов-на-Дону: Изд-во Ростов. ун-та, 1984.

13. Юдович В. И. Вибродинамика и виброгеометрия механических систем со связями// Усп. мех. - 2006. - 4, № 3. - C. $26-129$.

Хацкевич Владимир Львович

Военно-воздушная академия им. проф. Н. Е. Жуковского и Ю. А. Гагарина, Воронеж E-mail: vlkhats@mail.ru 